\title{
On the solid-liquid equilibrium behavior of fatty acids with ethanolamines
}

\author{
G.J. Maximo ${ }^{1}$, J.A.P. Coutinho ${ }^{2}$, M.C. Costa $^{3}$, and A.J.A. Meirelles ${ }^{1}$ \\ ${ }^{1}$ Laboratory ExTrAE, School of Food Engineering, University of Campinas, Campinas, Brazil \\ ${ }^{2}$ CICECO, Chemistry Department, University of Aveiro, Aveiro, Portugal \\ ${ }^{3}$ School of Applied Sciences, University of Campinas, Limeira, Brazil
}

Fatty acids and ethanolamines are commonly used in the synthesis of compounds that are mainly used in pharmaceutical and chemical industry as surfactant agent in addition to play a number of key roles in several other kinds of processes. Taking into account the formulation of mixtures with particular physicochemical properties for product and industrial processes design, information about systems' phase transitions is frequently required, although these data are yet scarce in the literature. In this work, the solid-liquid phase equilibrium (SLE) diagrams of four systems of monoethanolamine + oleic acid, monoethanolamine + stearic acid, diethanolamine + oleic acid and diethanolamine + stearic acid were determined. The temperature and enthalpy of the thermal transitions of the systems were evaluated by Differential Scanning Calorimetry (DSC) using a DSC8500 calorimeter (PerkinElmer, Waltham). The methodology was based in a cooling-heating cycle in order to evaluate the behavior of the crystallization and melting of the samples. Moreover, density, viscosity, surface tension and optical micrographs of some of the samples among the systems were determined/obtained in order to provide a best understanding of their rheological profile. The experimental results allowed the observation of different regions of solidliquid and solid-solid phase equilibrium from the diagrams obtained. These regions could be correlated with the physicochemical properties and optical micrographs showing that the systems presented particular temperatureand concentration-dependent phase behaviors. The results suggested that mixtures of fatty acids and ethanolamines presented interesting melting, crystallization and rheological properties for several applications in chemical processes and product formulation. 